\title{
Supply Voltage Compensation of Hot Wire Flow Measurement Device
}

\author{
Wang Haitao ${ }^{\mathrm{a}}$, Lang Xiaohua ${ }^{\mathrm{a}}$, Yang Haiping ${ }^{\mathrm{a}}, \mathrm{Li} \mathrm{Ye}^{\mathrm{b}}$, Yu Mengsun ${ }^{\mathrm{b}}$ \\ ${ }^{a}$ School of Control Science and Engineering Shandong University, Jinan, China \\ ${ }^{b}$ Aviation Medical Institution, Beijing, China
}

\begin{abstract}
Supply voltage compensation is important to keep the accuracy for hot wire/hot film gas flow sensors. A compensation experiment device is established, the two steps curve fitting is presented to determine the functional relations among flow sensor output and supply voltage, the coefficient of fitting polynomial is determined by Least Square method, thus eliminating the influence of supply voltage variations. After compensation, the error is reduced to $2 \%$. The algorithm is stabilized, easily to be fullfilled in microcontroller unit.
\end{abstract}

Index Terms: Two step curve fitting; supply voltage compensation; hot wire ;flow sensor

(C) 2011 Published by MECS Publisher. Selection and/or peer review under responsibility of the Research Association of Modern Education and Computer Science.

\section{Introduction}

The hot wire/film flow sensors are extensively applied in many fields, for example, ventilator, cardiopulmonary function device and respiration physiology measurement devices, having virtue of excellent sensitivity and accuracy; it is the perfect candidate for small range flow measurement. The operation theory of this sensor is as following: heating up the hot wire resistance with current, the gas flux take away a part of heat, whose quantity is proportional to the flow. The heat produced by current is determined by the current and resistance value. When the current is kept constant, the gas mass flow can be acquired by measuring the resistance, the above method is called Constant Current Method, another method named Constant Temperature Method, measures the current to calculate flow while keeping the temperature (or resistance) of hot wire constant. The latter method has excellent dynamic performance and is the mainstream of hot wire flow sensor[1,2]. But the hot wire sensor has two deficiencies. The first is temperature drift , that is, in the condition of constant flow, the output of sensor vary along with the temperature change of gas; the second is that the output of sensor is influenced by the supply voltage, which especially deteriorate the measurement accuracy of sensors powered by battery. For the first error, several software compensation have been introduced[3,4]. For the second error, voltage regulator can be used to stabilize the power supply, but this increase the power consumption, reduce the life time of battery of portable flowmeter, so it is necessary to develop supply voltage compensation for sensor powered by battery, With the development of microcontroller technology integrating with soft compensation

* Corresponding author.

E-mail address:Wht206@163.com 
algorithm, the intelligence of flowmeter is enhanced and the error originating from supply voltage variation can be reduced.

Software compensation of nontarget variables includes many kinds of algorithms, e.g. radial basic function or back propagation neural network[5,6], support vector machine[7], two dimensional regression analysis[8]. Aiming at a model of hot wire sensor, compensation experiment device is established, microcontroller is used to collect the output of flow sensor and supply voltage, then two step curve fitting is used to compensate the flow data, lastly, an intelligent hot wire flow measurement system suitable for power supply of battery is designed.

\section{Supply Voltage Compensation Experiment Device and Curve Fitting Algorithm}

\section{A. Supply Voltage Compensation Experiment Device}

In order to determine the relationship between input and output of flow sensor, an experiment plant is created shown as figure 1. A constant direct current power supply the flow sensor, the supply voltage can consecutively vary in the range of $8-15 \mathrm{~V}$. The output of sensor is converted into digital data by microcontroller, the digital data can be uploaded into computer by serial interface, also is displayed on LCD screen. Air flow is provided by a high pressure gas vessel, then get across pressure reductor and needle valve in order, last across the hot wire sensor, at the same time, a high precision flowmeter is connected to hot wire sensor in series to calibrate the flow value. The calibrate flometer is TSI certifier FAplus.

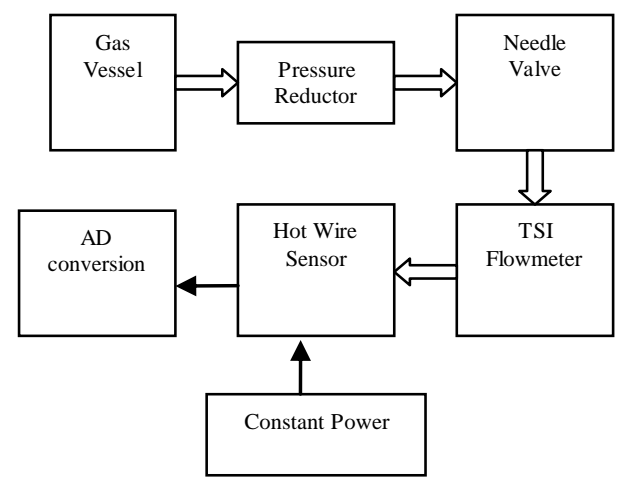

Fig 1. Supply voltage compensation experiment device

Table 1 Output Of Sensor Before Compensation

\begin{tabular}{|c|c|c|c|c|}
\hline \multirow{2}{*}{$\mathrm{F} / \mathrm{ml} / \mathrm{s}$} & \multicolumn{4}{|c|}{$V_{j} / \mathrm{V}$} \\
\cline { 2 - 5 } & 8.15 & 10 & 12 & 14 \\
\cline { 2 - 5 } & \multicolumn{3}{|c|}{$V_{A D C}$} \\
\hline 100 & 124 & 152 & 180 & 212 \\
\hline 200 & 232 & 286 & 339 & 401 \\
\hline 300 & 322 & 396 & 470 & 551 \\
\hline 400 & 400 & 495 & 586 & 689 \\
\hline 500 & 473 & 580 & 690 & 789 \\
\hline 600 & 532 & 644 & 778 & 856 \\
\hline
\end{tabular}

B. Curve Fitting Algorithm

The relationship between input(flow value) and output(the output voltage value after analog to digital converter ) vary with the change of supply voltage. A group of one-dimension polynomial, called characteristic 
curve, can be used to fit the above relationship. In the condition of variational supply voltage, the coefficients of polynomial also vary, which can be fitted by another group of one-dimension polynomial. The coefficients of two groups of curves can be solved via calibration data including TSI flow value, digital output of sensor and supply voltage value. The procedure need to find two groups equations respectively, so it is called two step curve fitting method, which is described in details as following.

First, establish characteristic curve cubic polynomials in condition of supply voltage ${ }^{V_{j}}$, as in (1), the digital output $\left(V_{A D C}\right)$ of sensor is treated as independent variable, flow value $\left({ }^{F_{j}}\right)$ from TSI flowmeter as dependent variable,

$$
F_{j}=a_{0 j}+a_{1 j} V_{A D C}+a_{2 j} V_{A D C}^{2}+a_{3 j} V_{A D C}^{3}
$$

Then Least Square method is used to find characteristic curve coefficients $\left\{a_{i j}\right\}, i=0,1,2,3$, the coefficients is related to supply voltage $V_{j}$.

Secondly, coefficients curve 2 degree polynomials are created to fit the characteristic curve coefficients by $V$ as in (2),

$$
a_{i}=A_{i}+B_{i} V_{j}+C_{i} V_{j}^{2}
$$

The solution method of $\left\{A_{i}, B_{i}, C_{i}\right\}$ is the same as in the first step, i.e. Least Square method. After determining the two groups of polynomials, the flow value can be acquired for arbitrary supply voltage.

\section{Result of Compensation Experiment and Discussion}

Before compensation, the input and output of hot wire sensor for various supply voltage are shown in table I . From the data, measurement error, up to $40 \%$, can be observed due to different supply voltage, even the real flow keeping constant. After compensation, the characteristic curve polynomials are used to compensate the voltage change influence on output. For instance, in the condition of $9 \mathrm{~V}$ and $13.5 \mathrm{~V}$ supply voltage, the characteristic curves are as follows[9]:

$$
\begin{aligned}
& F_{9 V}=-1.84607+0.75877 \cdot V_{A D C}-5.49652 \cdot V_{A D C}^{2} \\
& +9.02533 \cdot 10^{-7} \cdot V_{A D C}^{3} \\
& F_{13.5 V}=-1.19824+0.54716 \cdot V_{A D C}-2.89240 \cdot V_{A D C}^{2} \\
& +5.57873 \cdot 10^{-7} \cdot V_{A D C}^{3}
\end{aligned}
$$

After compensation, the output of sensor for supply voltage $9.0 \mathrm{~V}$ and $13.5 \mathrm{~V}$ is shown in table II, as can be seen, measurement error is reduced to $2 \%$ after compensation.

Table 1 Output For 9.0, 13.5V After Compensation

\begin{tabular}{|l|l|l|}
\hline $\mathrm{F} / \mathrm{ml} / \mathrm{s}$ & $\mathrm{Vj} / \mathrm{V}$ & \\
\hline & 9 & 13.5 \\
\hline 100 & 101 & 102 \\
\hline 200 & 202 & 201 \\
\hline 300 & 298 & 303 \\
\hline 400 & 396 & 397 \\
\hline 500 & 504 & 496 \\
\hline 600 & 606 & 595 \\
\hline
\end{tabular}




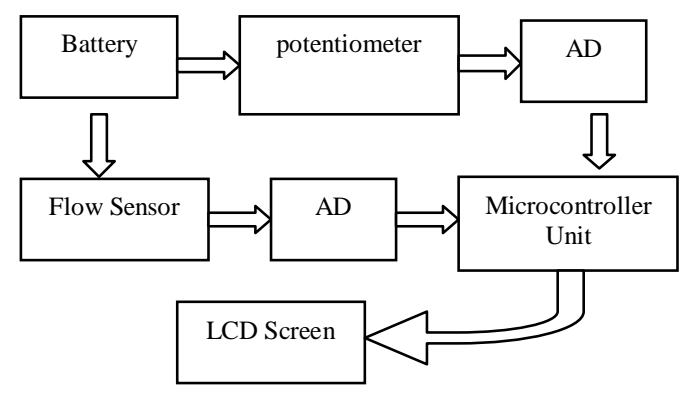

Fig 2 An intelligent flow measurement system

After development of compensation model, an intelligent flow measurement system powered by battery is set up, consisting of microcontroller unit(MCU), sensor and analog to digital converter, the electric circuit block is shown in Fig.2.

The characteristic and coefficient polynomials are stored in microcontroller, the battery voltage and output of sensor are sampled into the microcontroller, then the flow value is calculated from sampled data and fitting polynomials.

For portable flow sensor system, power dissipation is a key parameter, the software compensation method can greatly reduce the power dissipation as low dropout linear regulators is not required any more (in this system, the requirement power supply of the flow sensor is $8-15 \mathrm{~V}, 20 \mathrm{~mA}$, quite big compare to the MCU+ADC's $1 \mathrm{~mA}$ power dissipation). With respect to the order of fitting polynomials, it is not that the higher the order of polynomial, the smaller fitting error. The summed square of residuals produced from 4th order polynomial is nearly the same as ones done by the 3rd order polynomial, so it is desired to choose 3rd order. The function relation among flow, output of sensor and supply voltage also can be modeled with two-dimensional regression equation, but, with the same order, the coefficient matrix produced by two-dimensional regression has higher order, which lead to nonstable solution, even ill solution. Two step curve fitting adopts two groups of one-dimensional polynomial, resulting in lower scale of matrix when Least Square method is executed, the stabilization of solution is enhanced.

\section{Conclusion}

Two step curve fitting is presented to perform supply voltage, and hardware circuit is also designed. Data analyses show that measurement error is apparently reduced to $2 \%$ after compensation. The compensation algorithm is readily to be executed in microcontroller unit.

\section{References}

[1] Y.X. Su, J. Sheng, and G. W. Lang, "Flow measurement and testing," China Measurement Press,1992.(in Chinese)

[2] K.G.WU, "Frequency response of hot-film air mass flow sensors," Jouranl of Chang'an University,2005,25(5):pp.99-102.(in Chinese)

[3] J. P.Liu and G. L. Shi, "Hot wire mass flowmeter with an environment temperature compensation circuit," Chinese Journal of Sensors and Acurators,2006, 19(4):pp.1110-1116. (in Chinese)

[4] W. G.Zhao, Z.H.Song, and Z.W.Huang, "Thermal gas flow measurement based on single sensors," Chinese Journal of Sensors and Acurators,2009,22(3):pp.442-446. (in Chinese)

[5] X.J.Zhang, M.L.Zhang, and X.H.Li, "Temperature compensation of the CO electrochemical gas sensor based on RBF neural network," Chinese Journal of Sensors and Acurators, 2009,22(1):pp.11-14. (in Chinese) 
[6] G.Y.L, Y.C.Sun,and G.F.Pan, "Information fussion of pressure sensor based on BP network," Chinese Journal of Science Instrument,2005,26(2):pp.168-171. (in Chinese)

[7] W.F.Liang, X.D.Wang, and P.E.Liang, "Pressure sensor temperature compensation based on least squares support vector machine," Chinese Journal of Science Instrument,2007,28(12):pp.2235-2238. (in Chinese)

[8] M.Gao, W.K.Lu, and R.T.Sun, "Temperature compensation of hall current sensor based on two-dimensional regression analysis," Journal of Electronic Measurement and Instrument, 2009,23(2):pp.100-104. (in Chinese)

[9] Q.Li, L.Liang, and ZH.Liu, "Intelligent pressure sensor system with temperature compensation," Chinese Journal of Science Instrument, 2008, 29(9):pp.1934-1938. (in Chinese) 